\title{
Síndromes de hipersensibilidad inducidos por medicamentos en las unidades de cuidados intensivos
}

Drug-induced hipersensitivity syndromes in intensive care units

\author{
Marcela Cardona ${ }^{1}$, Luisa Fernanda Galindo², Diego Andrés Díaz-Guío³ \\ 1. Médica general, Vital Care, Centro de Simulación Clínica, grupo EdSimC, Armenia, Colombia \\ 2. Médica dermatóloga, Dermobog, Bogotá, D.C., Colombia \\ 3. Médico intensivista, Ph.D. (c) en Educación, Clínica Central del Quindío, CEO Vital Care, Centro de Simulación, Armenia, Colombia
}

\section{RESUMEN}

Las enfermedades cutáneas desencadenadas por medicamentos son comunes y, en su mayoría, no fatales; sin embargo, algunas de ellas menos frecuentes, son potencialmente mortales. Se destaca el espectro del síndrome de Stevens-Johnson/ necrólisis epidérmica tóxica y el síndrome de hipersensibilidad a medicamentos con eosinofilia y síntomas sistémicos.

Al ser enfermedades de baja incidencia, es común que el personal de cuidados intensivos no esté familiarizado con su presentación, diagnóstico y tratamiento, lo que puede asociarse con demoras en la atención adecuada y con impacto en la morbimortalidad.

En esta revisión narrativa se abordan estas entidades clínicas y se describe su asociación con medicamentos de uso frecuente en las unidades de cuidados intensivos; además, se tratan la fisiopatología, la presentación clínica y el tratamiento.

PALABRAS CLAVE: síndrome de Stevens-Johnson, síndrome de hipersensibilidad a medicamentos, cuidados críticos, preparaciones farmacéuticas, unidad de cuidados intensivos, erupciones cutáneas.

\section{SUMMARY}

Cutaneous diseases triggered by medications are common and usually harmless, but some of the less frequent variations can be life threatening. Some of the diseases in this category are the spectrum of Stevens-Johnson syndrome/ toxic epidermal necrolysis and drug reaction with eosinophilia and systemic symptoms syndrome.

The rare occurrence of these diseases has led to low levels of knowledge on its clinical presentation, diagnosis and treatment by medical staff on intensive care units, which can be associated with attention delays and negative impact on morbimortality.
Correspondencia:

Diego Andrés Díaz-Guío

Email:

andres.diaz@vitalcare.co

Recibido:12/03/18

Aceptado: 19/07/18

Conflictos de interés:

No se reportan conflictos de interés.

\section{Financiación:}

Ninguna 
This article reviews the above conditions, and describes its association with medications frequently used on intensive care units, as well as its pathophysiology, clinical presentation and treatment.

KEY WORDS: Stevens-Johnson syndrome, drug hypersensitivity syndrome, critical care, pharmaceutical preparations, intensive care units.

\section{INTRODUCCIÓN}

Muchos de los medicamentos utilizados en las unidades de cuidados intensivos pueden generar reacciones secundarias que, con frecuencia, son manifestaciones cutáneas, en su mayoría leves ${ }^{(1)}$. Sin embargo, ocasionalmente su presentación es potencialmente mortal, como es el caso del síndrome de Stevens-Johnson (SSJ), la necrólisis epidérmica tóxica (NET) y el síndrome de hipersensibilidad a medicamentos con eosinofilia y síntomas sistémicos (Drug Reaction with Eosinophilia and Systemic Symptoms, DRESS).

Se hizo una revisión narrativa de la literatura con el fin de establecer el conocimiento actual sobre las reacciones cutáneas graves generadas por medicamentos de uso frecuente en las unidades de cuidados intensivos, sus características de presentación, diagnóstico y tratamiento.

Se buscó en la literatura en español e inglés, en PubMed y en las bases de datos de los autores, con las palabras clave en los términos MeSH "Stevens-Johnson Syndrome”, “Critical Care”, “Intensive Care Units” y la combinación de los términos MeSH de los medicamentos implicados en la enfermedad, y se encontraron 991 artículos. Para el síndrome DRESS se usaron los términos MeSH "Drug Hypersensitivity Syndrome" y "Pharmaceutical Preparations", y el término MeSH de cada uno de los medicamentos implicados en la enfermedad, y se encontraron 255 artículos. En total, de los 1.246 artículos encontrados, se seleccionaron 118 según los siguientes criterios: tener resumen, ser sobre pacientes adultos y tener una relación directa con el objeto de estudio. De estos, se incluyeron 54 que se consideraron pertinentes para hacer esta revisión.

\section{SÍNDROME DE STEVENS- JOHNSON Y NECRÓLISIS EPIDÉRMICA TÓXICA}

El sindrome de Stevens-Johnson fue descrito por primera vez en 1922 por el cirujano Albert Stevens y el pe- diatra Frank Johnson ${ }^{(2,3)}$. En 1956, Alan Lyell, dermatólogo francés, describió la necrólisis epidérmica tóxica (4).

Actualmente, se considera que ambas son espectros de una misma enfermedad y reciben su nombre según el área de superficie corporal (ASC) comprometida: menos del $10 \%$ en el primer caso, más del $30 \%$ en el segundo caso, y de 10 a $30 \%$ en la superposición.

La incidencia anual de la necrólisis epidérmica tóxica oscila entre 0,4 y 1,86 casos por millón de habitantes ${ }^{(5,6)}$, y tiene una mortalidad de 10 a $45 \%{ }^{(7,8)}$. El síndrome de Stevens-Johnson es más frecuente, 1 a 6 casos al año por millón de habitantes, y su mortalidad es del $5 \%{ }^{(5)}$. La superposición de las dos enfermedades alcanza una mortalidad cercana al $6 \%{ }^{(8)}$.

Badia, et al., hicieron un estudio de 1.429 pacientes hospitalizados en una unidad de cuidados intensivos durante un periodo de tres años, 133 presentaron al menos una enfermedad dermatológica, entre los cuales se presentó únicamente un caso de necrólisis epidérmica tóxica ${ }^{(9)}$. Peña realizó un estudio en una unidad de cuidados intensivos Colombiana, durante un periodo de 7 meses; ingresaron 186 pacientes y de estos se documentaron dos casos de reacción secundaria a medicamentos, dentro de ellos un caso de SSJ ${ }^{(10)}$.

En la literatura científica se describen factores de riesgo que pueden aumentar la incidencia y gravedad del cuadro clínico, como la infección por el virus de la inmunodeficiencia humana (VIH), la tuberculosis, las neoplasias malignas, el lupus eritematoso sistémico y la administración rápida de medicamentos en grandes dosis; también, factores físicos como la radioterapia y la exposición a radiación ultravioleta, la pertenencia a algunas etnias y la predisposición genética según el antígeno leucocitario humano (Human Leukocyte Antigen, HLA) ${ }^{(6,10-12)}$

\section{ETIOLOGÍA Y FISIOPATOLOGÍA}

Inicialmente, se consideró que el conjunto del síndrome de Stevens-Johnson y la necrólisis epidérmica tóxica hacía parte de la forma mayor del eritema multiforme, pero, en 1997, el francés Jean Claude Roujeau y su grupo establecieron que estas enfermedades eran dos entidades diferentes, cada una con diferentes manifestaciones ${ }^{(11)}$. Por ejemplo, el conjunto del síndrome de Stevens-Johnson y la necrólisis epidérmica tóxica se presenta con aumento sérico de la interleucina 13 (IL13) y lesiones en parches o placas en diana atípicas, mientras que las lesiones del eritema multiforme son en diana típicas o atípicas levantadas ${ }^{(11)}$.

El espectro clínico del síndrome de Stevens-Johnson 
y la necrólisis epidérmica tóxica es considerado una reacción de hipersensibilidad retardada o de tipo IV, desencadenada principalmente por medicamentos, aunque también se ha sabido que infecciones por Mycoplasma pneumoniae, virus herpes, Escherichia coli y Klebsiella spp. entre otras, pueden desencadenar la enfermedad con menor frecuencia ${ }^{(12-14)}$.

Los medicamentos de uso frecuente en la unidad de cuidados intensivos que más se han asociado con esta enfermedad son: los anticonvulsivantes aromáticos, como la carbamazepina y la fenitoína, y el trimetoprim-sulfametoxazol; estos son los que deben generar la primera sospecha ${ }^{(15-17)}$. Sin embargo, otros medicamentos como la vancomicina, los medios de contraste, la piperacilina-tazobactam, las cefalosporinas, el levetiracetam y el valproato de sodio, entre otros, también pueden desencadenarla ${ }^{(7,14,18)}$.

Entre las diferentes teorías se encuentra la de la activación de los linfocitos T CD8 (LT-CD8) y las células asesinas naturales, mediante la expresión del medicamento como antígeno en los HLA de las células presentadoras de antígeno; esto lleva a la producción de diferentes moléculas que culminan en la apoptosis de queratinocitos, haciendo que la epidermis se desprenda de la dermis ${ }^{\left({ }^{19}\right)}$. La principal molécula implicada en la enfermedad es la granulisina, que es un mediador de muerte celular producido por los LT-CD8 $(7,19,20)$. Por medio del estudio del contenido de las ampollas, se ha evidenciado que, a mayor concentración de este mediador, mayor la gravedad de la enfermedad. Incluso, se ha aplicado esta molécula a la piel de ratones, generando ampollas y desprendimiento de la piel ${ }^{(19,21)}$.

Otras moléculas asociadas son el factor de necrosis tumoral alfa (TNF- $\alpha$ ), el interferón gamma (IFN- $\gamma$ ), la granzima B, las perforinas, interacción Fas/Fas ligando, IL-2, IL-5, IL-6, IL-10, IL-12, IL-13, IL-15, IL-18 y las quimiocinas, entre cuyas funciones se incluyen aumentar la reacción inflamatoria, la expresión del HLA y la apoptosis de las células blanco de la enfermedad, los queratinocitos ${ }^{(19,22)}$.

Finalmente, el desprendimiento de la epidermis lleva a una insuficiencia cutánea aguda pues la piel no puede cumplir sus funciones fisiológicas; se aumenta la pérdida transepidérmica de agua, se absorben sustancias de manera percutánea, se pierde la protección contra microrganismos y rayos ultravioleta, y se altera la capacidad de termorregulación; además, se genera redistribución del gasto cardiaco por vasodilatación, hipotermia que aumenta la tasa metabólica y, por tanto, produce hiperglucemia, alteraciones electrolíticas, anemia e hipoproteinemia. En consecuencia, las causas de muerte son deshidratación, sepsis, síndrome de dificultad respiratoria aguda, neumonitis y falla multiorgánica, entre otras ${ }^{(19,22,23)}$.

Se ha encontrado que el HLA presente en algunas etnias, como la han de China, favorece el desarrollo de la enfermedad, como es el caso del HLA-B*15:02 con la carbamazepina y la fenitoína ${ }^{(6)}$.

\section{CLÍNICA}

Usualmente, el cuadro clínico se inicia entre 4 y 28 días después de iniciado el medicamento, en promedio 14 días, pero una nueva exposición puede preceder los síntomas solo 48 horas ${ }^{(24,25)}$. Se divide en dos fases.

Fase aguda: suele empezar con síntomas inespecíficos, como fiebre, astenia, adinamia y disfagia, lo que puede retrasar el diagnóstico y el retiro del medicamento causante $^{(22)}$. Hay dolor urente en la piel y en las mucosas (cavidad oral, conjuntiva, genitales).

Entre uno y tres días después, empieza la erupción cutánea con lesiones en diana atípicas, planas, máculas eritematosas, purpúricas y mal definidas, o eritema difuso, con dolor a la palpación desproporcionado con respecto a las lesiones visibles. Afecta principalmente la región anterior del tronco, la cara y la región proximal de extremidades, y se generaliza simétricamente. Las lesiones pueden confluir hasta llegar a afectar grandes áreas de superficie corporal ${ }^{(6,11,22)}$. El desprendimiento de la epidermis se inicia con ampollas flácidas. Cuando estas no están presentes, se pueden provocar ejerciendo presión tangencial sobre la piel afectada, lo cual genera el desprendimiento; esto se conoce como el signo de Nikolsky. Cabe resaltar que este también se encuentra en las enfermedades ampollosas autoinmunitarias ${ }^{(6)}$. La apariencia final al progresar la enfermedad, es similar a la de una quemadura térmica extensa (figura 1) ${ }^{(26)}$.

Las mucosas están comprometidas en el 90 \% de los casos; su afectación puede preceder o seguir al compromiso cutáneo ${ }^{(17,22)}$. Al romperse las ampollas de las mucosas, se cubren con una seudomembrana grisácea, lo cual dificulta la apertura ocular, la deglución y la micción. Muy rara vez se ve compromiso de la mucosa anal. La elevación de las transaminasas no se asocia con mayor gravedad del cuadro clínico. No es frecuente, pero se ha descrito la perforación intestinal $(6,19,22,27,28)$.

Se requiere asistencia respiratoria mecánica en el 25 $\%$ de los casos. Los principales factores de riesgo para requerir asistencia respiratoria son: bicarbonato sérico menor de $20 \mathrm{mEq} / \mathrm{L}$, nitrógeno ureico en sangre (Blood Urea Nitrogen, BUN) mayor de $28,08 \mathrm{mg} / \mathrm{dl}$, compro- 
miso de la superficie corporal mayor del $10 \%$, sobre todo si es mayor o igual a $30 \%$, más de 12.000 leucocitos por $\mathrm{mm}^{3}$, hemoglobina de menos de $8 \mathrm{mg} / \mathrm{dl} \mathrm{o}$ infiltrados pulmonares. La necesidad de asistencia respiratoria mecánica, se asocia con mayor desarrollo de complicaciones como choque, falla renal aguda que requiere hemodiálisis, neumonía y bacteriemia; además, con una mayor mortalidad ( $57 \%$ de los pacientes con dicha asistencia murieron en el hospital). Se recomienda prestar especial atención a los pacientes con signos que sugieran compromiso bronquial, como hipersecreción mucosa, disnea, hipoxemia o compromiso laríngeo ${ }^{(29)}$.

La gravedad de la enfermedad puede calcularse con la escala SCORTEN (Score of Toxic Epidermal Necrolysis). Consta de siete variables independientes de mortalidad (tabla 1) y cada una da un puntaje de 1; 0-1 puntos se relacionan con una mortalidad de 3,2 \% y, 5 o más puntos, con una mayor del $90 \%{ }^{(19,30)}$. Se recomienda calcular este puntaje en las primeras 24 horas de admisión al servicio ${ }^{(27)}$.

Fase crónica: en esta fase se hace un seguimiento en busca de las secuelas que se pueden desarrollar, como cambios de pigmentación en la piel, distrofias ungu- lares, sinequias oculares, compromiso de la agudeza visual, bronquiolitis obliterante o estenosis del canal vaginal, entre otras ${ }^{(19)}$.

\section{DIAGNÓSTICO}

No hay criterios diagnósticos universales aceptados para el conjunto del síndrome de Stevens-Johnson y la necrólisis epidérmica tóxica, y la histología no es específica para la enfermedad. A pesar de estas limitaciones, el diagnóstico se hace ante la presencia del cuadro clínico descrito, con historia sugestiva de exposición a medicamentos o enfermedad febril previa. Se debe interrogar sobre el inicio de los síntomas inespecíficos, dolor en la piel, medicamentos consumidos durante las últimas ocho semanas, y además, buscar las lesiones características de la enfermedad que rápidamente pueden unirse, y producir ampollas y desprendimiento de la piel (signo positivo de Nikolsky). Es importante buscar el compromiso de las mucosas, pues puede presentarse tempranamente ${ }^{(27)}$. En la biopsia de piel, se encuentra necrosis de queratinocitos con desprendimiento de la base de la epidermis ${ }^{(6)}$.

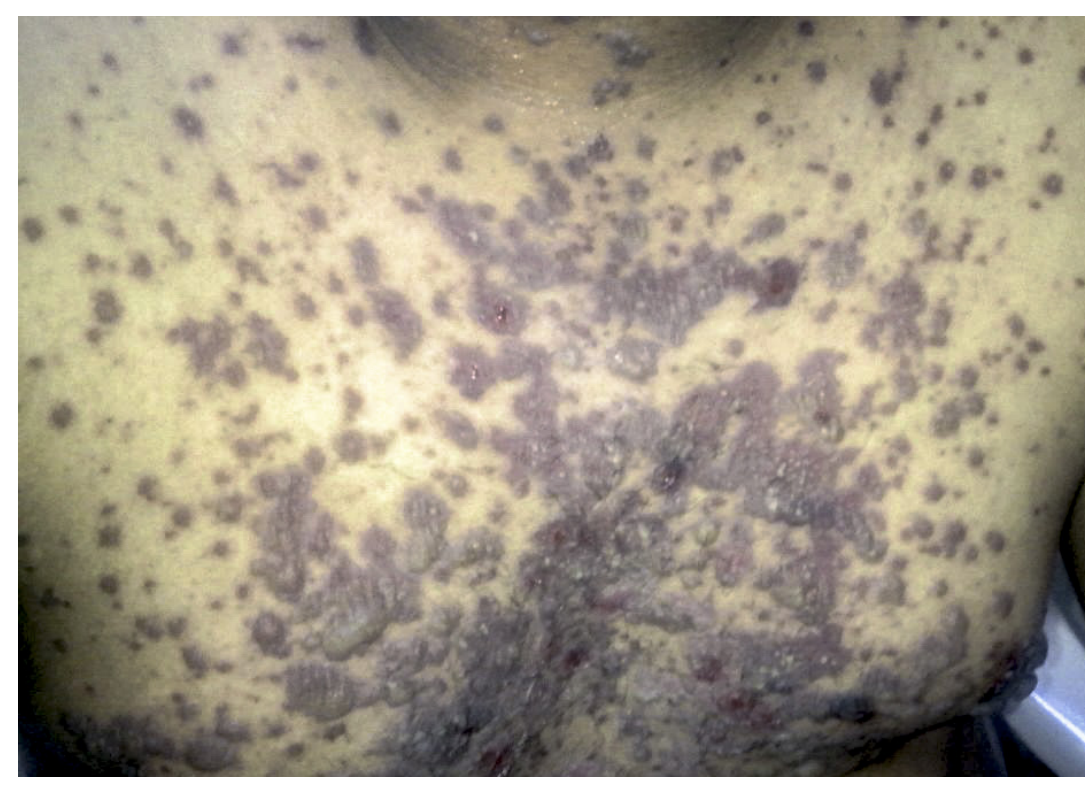

Figura 1. Ampollas flácidas en el tórax, con contenido serosanguinolento y coalescentes; se observan algunas ampollas escoriadas. Cortesía de Ángela Seidel 
Tabla 1. Puntaje para la necrólisis epidérmica tóxica, SCORTEN (Score of Toxic Epidermal Necrolysis)

\begin{tabular}{|c|c|c|}
\hline \multicolumn{3}{|c|}{ SCORTEN } \\
\hline Variable & Punto de corte & Puntaje \\
\hline Edad (años) & $>40$ & 1 \\
\hline Frecuencia cardiaca (latidos por minuto) & $>120$ & 1 \\
\hline $\begin{array}{l}\text { Cáncer o neoplasia maligna } \\
\text { hematológica }\end{array}$ & Presente & 1 \\
\hline Superficie corporal comprometida $>10 \%$ & Presente & 1 \\
\hline Nitrógeno ureico en sangre $(\mathrm{mg} / \mathrm{dl})$ & $>28$ & 1 \\
\hline Bicarbonato sérico (mEq/L) & $<20$ & 1 \\
\hline Glucemia (mg/dl) & $>252,22$ & 1 \\
\hline
\end{tabular}

\section{ABORDAJE Y TRATAMIENTO}

Abordaje inicial: el descontinuar el medicamento desencadenante es de vital importancia, hacerlo de manera temprana ha demostrado mejorar el pronóstico ${ }^{(6)}$. El manejo general de soporte es cardinal, y aún se requiere de estudios bien diseñados y reproducibles sobre el tratamiento sistémico para poder hacer recomendaciones basadas en evidencia de alta calidad.

Medidas de sostenimiento: los pacientes con compromiso de más del $10 \%$ de la superficie corporal, deben trasladarse de manera temprana (antes del séptimo día del ingreso hospitalario) a la unidad de cuidados intensivos o la unidad de quemados, donde deben tener un seguimiento estricto y ser tratados por personal entrenado ${ }^{(17,23)}$. La pérdida transdérmica de agua es importante y requiere de una adecuada reposición por medio de cristaloides; puede ser tres cuartas partes de la fórmula de Parkland o reanimación hídrica basada en metas, como mantener un gasto urinario entre 0,5 $\mathrm{y} 1$ $\mathrm{ml} /$ kg por hora $^{(23)}$.

Los catéteres venosos o arteriales deben insertarse en piel sana, mientras sea posible; los primeros deben cambiarse cada 48 horas. Se debe evitar movilizar bruscamente al paciente para no aumentar el área de desprendimiento de piel; además, hacer limpieza diaria de toda la piel (sana y enferma) con agua estéril, solución salina tibia o clorhexidina, evitando friccionarla ${ }^{(27)}$. La epidermis desprendida se puede dejar sobre la zona afectada pues sirve como apósito biológico ${ }^{(17)}$.

Es indispensable la evaluación oftalmológica al ingreso del paciente. Se deben aplicar gotas lubricantes, limpieza de detritos inflamatorios para evitar sinequias, corticoesteroide tópico y colirio antibiótico ${ }^{(27,31)}$. En la cavidad oral, es importante la limpieza diaria ${ }^{(27)}$. El área urogenital debe evaluarse diariamente; se puede considerar el cateterismo vesical para evitar estenosis uretrales ${ }^{(32)}$. En las mujeres, se han usado apósitos o dilatadores impregnados con esteroides en la vagina hasta que haya una recuperación completa para disminuir el riesgo de sinequias. A los hombres no circuncidados, se les debe evaluar diariamente la retractilidad del prepucio ${ }^{(27)}$.

En las guías del Reino Unido para el conjunto del síndrome de Stevens-Johnson y la necrólisis epidérmica tóxica en adultos (U.K. Guidelines for the Management of Stevens-Johnson Syndrome/Toxic Epidermal Necrolysis in Adults 2016), se recomienda usar la analgesia según el esquema de escalera de la Organización Mundial de la Salud, iniciando con acetaminofén; para el dolor leve a moderado, se dan opioides de tipo codeína, mientras que para el dolor moderado a grave, morfina ${ }^{(27,33)}$.

Se recomienda hacer profilaxis farmacológica para la trombosis, a menos que esté contraindicada, y brindar protección gástrica con un inhibidor de la bomba de protones ${ }^{(27)}$. El soporte nutricional se hace con dieta hipercalórica e hiperproteica ${ }^{(23)}$. Se debe mantener la temperatura de la habitación entre 30 y $32^{\circ} \mathrm{C}{ }^{(32)}$. Es ideal restringir el uso de antibióticos sistémicos profilácticos, a menos que haya manifestaciones clínicas de infección (19).

Tratamiento sistémico: hasta ahora no hay suficiente información para recomendar un tratamiento sistémico específico. De acuerdo con la fisiopatología, se han usado tratamientos inmunomoduladores para mitigar la extensión de la enfermedad, acelerar la recuperación y disminuir la mortalidad. Se han usado los esteroides sistémicos a dosis altas; sin embargo, hay discusión sobre 
su beneficio y sí es claro el riesgo de desarrollar sepsis e hiperglucemia, especialmente en pacientes críticos; por lo tanto, su uso es controversial ${ }^{\text {(34). }}$

El ligando Fas/Fas tiene un papel importante en el estímulo de la apoptosis de los queratinocitos; el bloqueo del receptor Fas con inmunoglobulina intravenosa en adultos, ha mostrado resultados contradictorios ${ }^{(17,35)}$. Algunos estudios muestran mayor beneficio al ser usada a dosis altas (>2 g/ $\mathrm{kg}$ ) con disminución del $59 \%$ de la mortalidad esperada ${ }^{(8)}$.

La ciclosporina, un medicamento de uso oncológico, inhibe la calcineurina y tiene un potente efecto inmunosupresor; ha tenido resultados positivos en la disminución de la mortalidad y la velocidad de reepitelización, aunque aún se requieren más estudios ${ }^{(17,27)}$.

En cuanto a los medicamentos anti-TNF- $\alpha$, la talidomida se asoció con mayor mortalidad. En un estudio con etanercept en dosis única de $50 \mathrm{mg}$ subcutáneos, ningún paciente murió, a pesar de tener una probabilidad promedio de mortalidad de 46,9 \% calculada con el SCORTEN ${ }^{(24)}$.

El glutatión reducido es un antioxidante de metabolitos de medicamentos; este podría estar disminuido en los queratinocitos del conjunto del síndrome de StevensJohnson y la necrólisis epidérmica tóxica desencadenado por algunos medicamentos, como el sulfametoxazol. Es por esto que se ha usado la $\mathrm{N}$-acetilcisteína, que es un precursor de este glutatión (36). Hay pocos estudios, pero se han conseguido resultados positivos, incluso, cuando no se ve mejoría clínica con la inmunoglobulina intravenosa ${ }^{(37)}$.

\section{SIINDROME DE HIPERSENSIBI- LIDAD A MEDICAMENTOS CON EOSINOFILIA Y SÍNTOMAS SISTÉMICOS}

Se trata de una enfermedad potencialmente mortal que se asocia con erupción cutánea máculo-papular, con compromiso de múltiples órganos internos. Su diagnóstico es difícil por la similitud del cuadro clínico con el de otras enfermedades más frecuentes, como la sepsis.

Fue descrita por primera vez con el uso de fenitoína y se conocía como síndrome de hipersensibilidad a la fenitoína; más adelante, se encontró que otros medicamentos podían desencadenar el mismo cuadro. En 1996, se propuso el nombre de síndrome de hipersensibilidad por medicamentos con eosinofilia y síntomas sistémicos (Drug Reaction with Eosinophilia and
Systemic Symptoms, DRESS) ${ }^{(38)}$. En 1998, un grupo japonés propuso el término alternativo de síndrome de hipersensibilidad inducido por medicamentos (Druginduced Hypersensitivity Syndrome. DiHS) después de encontrar la gran prevalencia de reactivación del virus herpes 6 (HHV6) en estos pacientes e incluyeron este hallazgo en sus propios criterios diagnósticos ${ }^{(39)}$.

Tiene una incidencia de 1 en 1.000 a 1 en 10.00o, dependiendo del medicamento al cual se exponga el paciente (40). En un periodo de 11 años, 23 unidades de cuidados intensivos en Francia reportaron 34 casos de DRESS, lo que muestra su poca incidencia ${ }^{(41)}$. La mortalidad es del $10 \%$, y depende del número de órganos comprometidos y de la gravedad de su compromiso; usualmente, la muerte se produce por lesión hepática con necrosis del parénquima. Puede generar falla hepática aguda y requerir trasplante hepático ${ }^{(38,42)}$.

\section{ETIOLOGÍA}

Se considera una reacción de hipersensibilidad tipo IV o tardía. Los principales medicamentos de uso frecuente en la unidad de cuidados intensivos que la desencadenan, son los anticonvulsivos aromáticos (carbamazepina, fenobarbital, fenitoína) y las sulfonamidas como el trimetoprim-sulfametoxazol. Se han descrito hasta 50 medicamentos que pueden ser los causantes (38, 40, 43); entre ellos, están antibióticos betalactámicos, cefalosporinas, fluoroquinolonas, ácido valproico y vancomicina (tabla 2) ${ }^{(42,44-49)}$.

Miyasu, et al., describieron el caso de un paciente con osteosíntesis de fémur y necrosis de la piel, en el que se detectó infección por Staphylococcus aureus resistente a la meticilina, por lo cual requirió tratamiento con vancomicina. En el día 18 de la antibioticoterapia desarrolló eritema en los miembros superiores y fiebre persistente. En el día 29, presentó eosinofilia de más de $700 / \mathrm{mm}^{3}$ y erupción cutánea extensa con edema palpebral, por lo que hicieron el diagnóstico de síndrome DRESS y suspendieron el medicamento. Se requirieron 11 días desde el inicio de los síntomas para hacer el diagnóstico y retirar el medicamento desencadenante, lo cual indica que es un diagnóstico difícil que siempre requiere de la sospecha clínica ${ }^{(48)}$.

El diagnóstico se hace aún más difícil cuando el cuadro clínico se inicia en la unidad de cuidados intensivos, debido a que la mayoría de pacientes reciben múltiples medicamentos. Moriceau, et al., informaron de una paciente de 53 años que recibía diferentes antibióticos, entre ellos, vancomicina, piperacilina-tazobactam y amikacina. Después del diagnóstico de síndrome DRESS, se suspendieron los antibióticos y se obtuvo 
Tabla 2. Medicamentos desencadenantes del síndrome DRESS (42,44-49)

\begin{tabular}{ll}
\hline Amoxicilina & Fenobarbital \\
\hline Antiinflamatorios no esteroideos & Lamotrigina \\
\hline Antituberculosos & Levetiracetam \\
\hline Alopurinol & Minociclina \\
\hline Antirretrovirales (abacavir, nevirapina) & Moxifloxacino \\
\hline Carbamazepina & Oxcarbazepina \\
\hline Cefalosporinas & Sulfonamidas \\
\hline Dapsona & Vancomicina \\
\hline Fenitoína & Teicoplanina \\
\hline
\end{tabular}

mejoría; se reintrodujo la piperacilina-tazobactam, pues se pensó que el causante era la vancomicina. Al reiniciarlo, la paciente se vio muy comprometida clínica y paraclínicamente, llegando a la aplasia medular (50).

Se ha descrito que los pacientes que desarrollan el síndrome DRESS por anticonvulsivantes y alopurinol, desarrollan con mayor frecuencia un estado de choque y, por lo tanto, presentan mayor mortalidad ${ }^{(41)}$.

\section{FISIOPATOLOGÍA}

Aún es poco claro el proceso fisiopatológico de este síndrome. Se han encontrado varias características que demuestran que se trata de una reacción de hipersensibilidad, como son su reproducibilidad con pruebas cutáneas, la reaparición de la enfermedad al haber una nueva exposición al medicamento desencadenante, y la necesidad de sensibilización previa.

Se sabe que hay una inmunosupresión en las primeras dos semanas del exantema, ya que hay predominio de linfocitos T reguladores. Se cree que estas células albergan ADN del virus del herpes (HHV), produciéndose reactivación de la infección viral durante la expansión clonal.

Además, se ha encontrado disminución de linfocitos B y, por lo tanto, de las inmunoglobulinas IgA, IgG e IgM. Durante ese periodo, se genera la mayor afección orgánica, principalmente hepática, con elevación de las transaminasas hasta la falla hepática. A medida que la función hepática regresa a lo normal, se observa aumento de los eosinófilos en sangre, posiblemente, por la presencia de IL-5 y eotaxina; se disminuye el número de linfocitos $\mathrm{T}$ reguladores y aumenta el de linfocitos T efectores ${ }^{(38,39,45,51)}$.
Los HLA, al igual que en el conjunto del síndrome de Stevens-Johnson y la necrólisis epidérmica tóxica, hacen que ciertas etnias sean más propensas ante algunos medicamentos específicos. Tal es caso del HLAB58:01, que predispone al desarrollo de DRESS y conjunto del síndrome de Stevens-Johnson y la necrólisis epidérmica tóxica ante el uso de alopurinol, y el HLAB31:01 que lo hace al desarrollo de DRESS con el uso de carbamazepina en japoneses y la población han de China ${ }^{(38,39)}$.

\section{CLÍNICA}

El síndrome DRESS tiene un periodo de latencia típico de dos a seis semanas después de iniciar la administración del medicamento causante ${ }^{(45)}$. Aunque no es usual que se inicie durante las primeras dos semanas ${ }^{(39)}$, algunos reportan que puede ocurrir desde los primeros 7 días hasta los 90 días después de la exposición ${ }^{(44)}$. Puede estar precedido por síntomas de infección de las vías respiratorias superiores y fiebre, que puede estar presentes días o semanas antes de la aparición de la erupción cutánea ${ }^{(39)}$. El compromiso de la piel es, típicamente, una erupción cutánea máculo-papular eritematosa, difusa y pruriginosa, que se inicia en la cara y en el tercio superior del tronco; posteriormente, evoluciona en dirección cefalo-caudal (39). En el proceso de recuperación, se produce descamación. En ocasiones, puede comprometer más del $90 \%$ de la superficie corporal y convertirse en una dermatitis exfoliativa (figura 2) ${ }^{(47)}$. Usualmente, hay importante edema periorbitario y facial $(38,39,44,47)$. Raras veces se producen ampollas, aunque Borok describió un caso de DRESS que cursó con ampollas, específicamente, sobre el área de un tatuaje que había sido realizado 10 años antes ${ }^{(52)}$. Puede 
comprometer las mucosas con menor gravedad que el conjunto del síndrome de Stevens-Johnson y la necrólisis epidérmica tóxica, y cursar con queilitis, eritema faríngeo, hipertrofia amigdalina, y edema bilateral de las glándulas salivales con xerostomía que puede dificultar la ingestión de alimentos ${ }^{38,39,51)}$.

El $75 \%$ de los pacientes presenta linfadenopatías dolorosas, principalmente cervicales, que histológicamente pueden presentar un patrón benigno o de seudolinfoma ${ }^{(38)}$. La leucopenia precede en muchas ocasiones a la linfocitosis y a la eosinofilia. Esta última está presente en $30 \%$ de pacientes y juega un papel importante en la lesión de los órganos. En un estudio retrospectivo de 26 pacientes, Sasidharanpillai, et al., encontraron que la mayor gravedad está asociada con la presencia de linfocitos atípicos ${ }^{(44)}$.

El órgano más comprometido es el hígado. Son frecuentes la elevación de las transaminasas, la hepatitis y la falla hepática por necrosis del parénquima, haciendo necesario el trasplante hepático ${ }^{(45)}$.

El riñón también se afecta con frecuencia. Hay elevación del nitrógeno ureico en la sangre y de la creatinina sérica, y puede llegar a nefritis intersticial, falla renal aguda y necesidad de diálisis ${ }^{(38,41,44)}$. La función respiratoria puede estar comprometida, con disnea, tos seca y neumonitis intersticial aguda. Los pacientes que desarrollan el síndrome de dificultad respiratoria aguda, requieren asistencia respiratoria mecánica invasiva inmediata ${ }^{\left({ }^{8}\right)}$ y tienen mayor riesgo de desarrollar secuelas.

Puede haber compromiso cardiaco. Se han descrito dos tipos: miocarditis por hipersensibilidad y miocarditis eosinofílica necrosante aguda. La miocarditis por hipersensibilidad suele ser de resolución espontánea; la eosinofílica necrosante aguda es de mal pronóstico, con una mortalidad de hasta el $50 \%$ (38). Usualmente, esta última cursa con dolor torácico, disnea, taquicardia sinusal, congestión pulmonar, disfunción del ventrículo izquierdo, y elevación de la creatincinasa

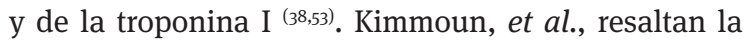
importancia de considerar el síndrome DRESS en el diagnóstico diferencial, en los pacientes en estado de choque y sin infección comprobada, pues frecuentemente requieren medicamentos vasoactivos y asistencia respiratoria mecánica ${ }^{(41)}$.

El compromiso neurológico, el gastrointestinal y el endocrino, son poco frecuentes ${ }^{(38)}$.

\section{DIAGNÓSTICO}

El diagnóstico es difícil debido al cuadro clínico inespecífico y a la falta de conocimiento de la enfermedad

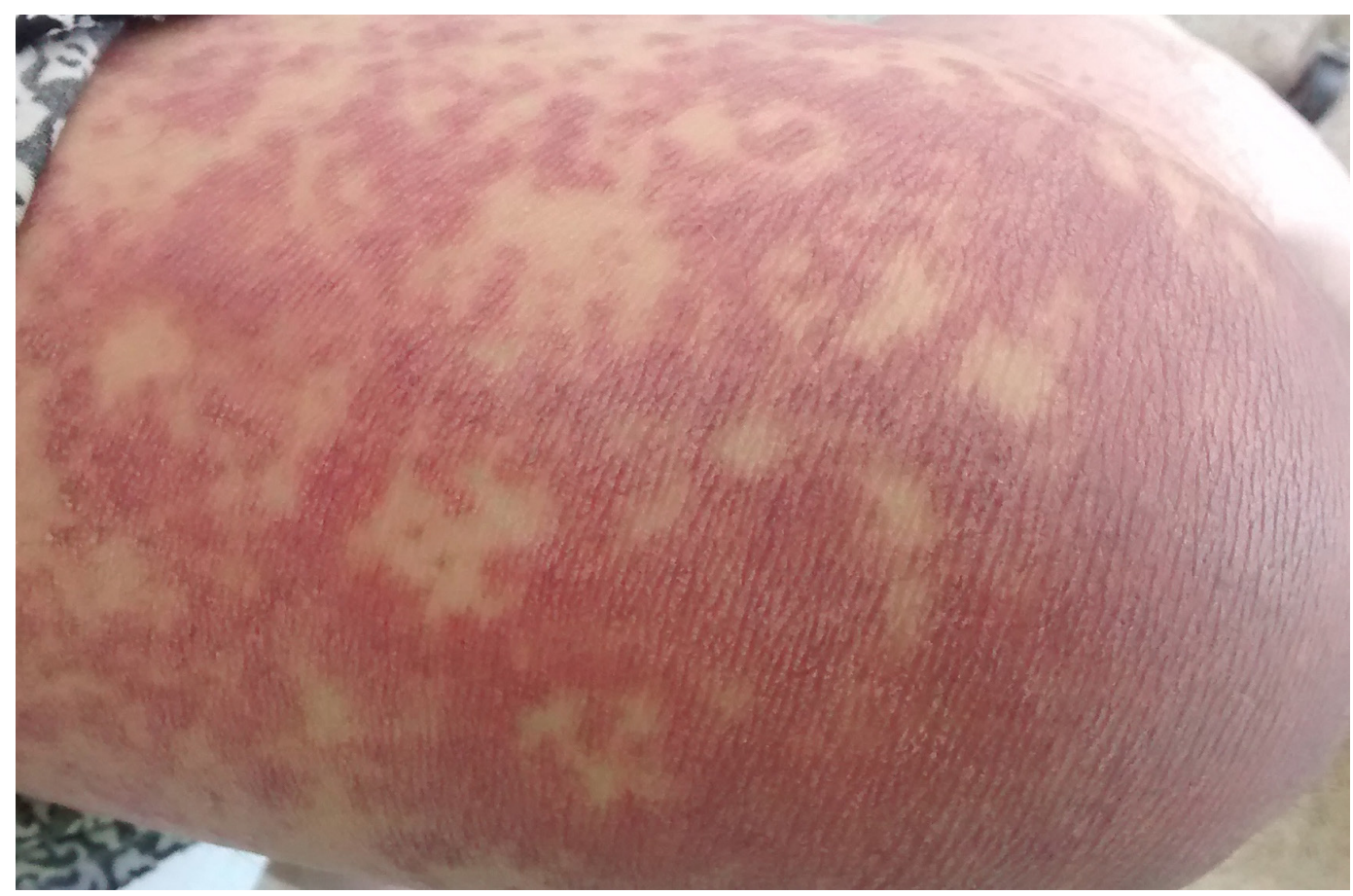

Figura 2. Máculas eritemato-violáceas, de bordes mal definidos, irregulares, con islas de piel sana. Cortesía de Ángela Seidel 
por gran parte del personal de salud. Se debe tener mucha sospecha, sobre todo al usar los medicamentos que tienen mayor asociación con el desarrollo de la enfermedad. Es importante descartar otras causas graves, como infección, enfermedades autoinmunitarias y neoplasias.

Entre los exámenes de laboratorio, es importante solicitar: hemograma, pruebas de función hepática, proteínas en orina de 24 horas y conteo de eosinófilos en orina, creatin-fosfo-cinasa, lactato deshidrogenasa (LDH), ferritina, triglicéridos, calcio, hormona paratiroidea, hormona estimulante de tiroides, glucemia, tiempo de protrombina, tiempo de tromboplastina, lipasa, proteína $\mathrm{C}$ reactiva, electroforesis de proteínas, reacción en cadena de polimerasa cuantitativa para HHV-6, HHV-7, virus de Epstein-Barr y citomegalovirus, hemocultivos, anticuerpos antinucleares ${ }^{(54)} \mathrm{y}$ el extendido de sangre periférico para detectar leucocitos atípicos $^{(47,49)}$.

Aún no se han estandarizado los criterios diagnósticos, pero se usan con mayor frecuencia los del Registry of Severe Cutaneous Adverse Reaction (RegiSCAR) (tabla 3) ${ }^{(38)}$ y los del Japanese Research Committee on Severe Cutaneous Adverse Reaction (J-SCAR) (tabla 4) ${ }^{(38,39)}$. Su principal diferencia es que los criterios japoneses incluyen la reactivación del HHV-6 ${ }^{(39)}$.

Se han encontrado factores de mal pronóstico, como la frecuencia cardiaca mayor de 90 latidos por minuto, leucocitosis mayor de $12.000 / \mathrm{mm}^{3} \mathrm{y}$ frecuencia respira- toria mayor de 20 por minuto al ingreso hospitalario; además, coagulopatía y sangrado digestivo en los primeros 30 días ${ }^{(55)}$, falla renal, falla respiratoria, lesión miocárdica o alteración del estado mental. También, se ha visto que la elevación de las transaminasas cinco veces por encima del límite superior normal, puede indicar necrosis hepática ${ }^{(47)}$.

Es muy importante identificar el medicamento que desencadenó el síndrome DRESS. Usualmente, esto es difícil en la unidad de cuidados intensivos. Dos pruebas pueden ayudar a identificarlo, pero de manera tardía: la prueba del parche y la prueba de transformación linfocitaria. Ambas aportan información cuando son positivas. El resultado negativo no descarta la relación del medicamento con la enfermedad. Se recomienda hacer la prueba del parche dos a seis meses después de la recuperación de los síntomas y la prueba de transformación linfocitaria se debe hacer cinco a ocho semanas después de iniciado el cuadro clínico ${ }^{(39,51,54)}$.

\section{TRATAMIENTO}

El tratamiento de referencia es descontinuar inmediatamente el medicamento culpable e iniciar el uso de corticoesteroides sistémicos. Usualmente, se da una dosis diaria de $1 \mathrm{mg} / \mathrm{kg}$ de prednisolona o sus equivalentes, la cual se va disminuyendo paulatinamente en un periodo aproximado de tres a seis meses para evitar recaídas.

Tabla 3. Registry of Severe Cutaneous Adverse Reactions (RegiSCAR)

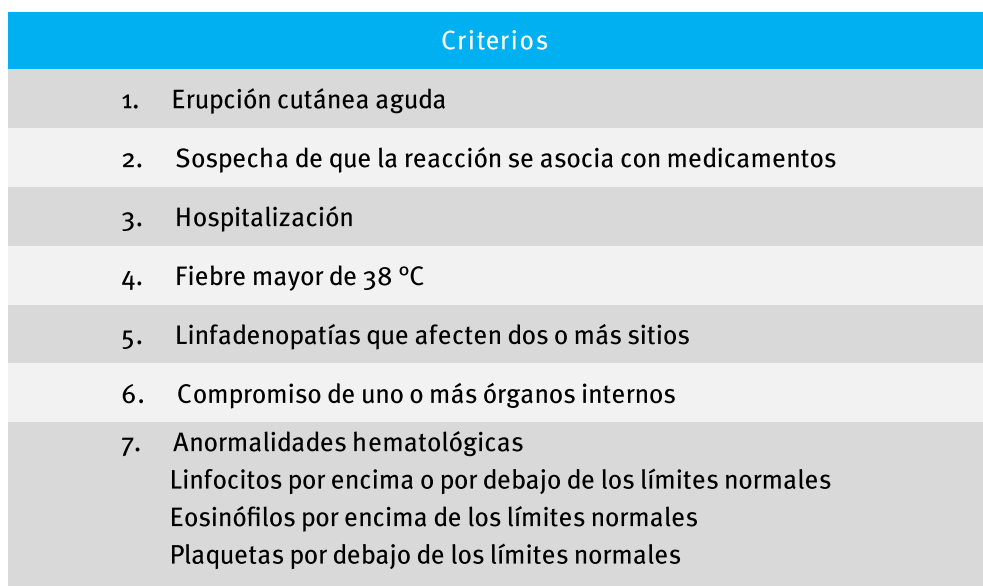

Nota: Para hacer el diagnóstico de DRESS, deben estar presentes el punto 1, 2 y 3, y tres de los puntos $4,5,6$ y $7^{(38)}$. 
Tabla 4. Criterios del Japanese Research Committee on Severe Cutaneous Adverse Reaction (J-SCAR)

\section{Criterios del Japanese Research Committee on Severe Cutaneous Adverse Reaction (J-SCAR)}

1. Erupción cutánea máculo-papular que se desarrolla después de más de tres semanas de iniciado el medicamento agresor

2. Síntomas prolongados después de descontinuar el medicamento agresor

3. Fiebre $>38^{\circ} \mathrm{C}$

4. Alteración de las pruebas hepáticas (ALT\100 U/L) o compromiso de algún otro órgano

5. Anormalidades leucocitarias $(\geq 1)$ Leucocitosis $>11 \times 109 / \mathrm{L}$ Linfocitos atípicos $>5 \%$ Eosinofilia $\mathbf{1 1 , 5}$ X109/L

6. Linfadenopatías

7. Reactivación del HHV-6

Nota: los criterios del J-SCAR incluyen el síndrome de hipersensibilidad inducido por medicamentos, DiHS (Drug-induced Hypersensitivity Syndrome). Un cuadro clínico se considera típico de DiHS, cuando cumple con los siete criterios, y atípico, cuando cumple solo cinco criterios ${ }^{(38,39)}$.

En la fase inicial, se brindan medidas de soporte, antipiréticos y esteroides tópicos para aliviar los síntomas locales.

Se recomienda hacer una búsqueda activa de lesión de órganos dos veces por semana, mediante pruebas de función renal y hepática, hemoleucograma, LDH y otras pruebas específicas para cada órgano.

En caso de no haber mejoría con la prednisolona oral, se puede suministrar metilprednisolona a dosis de 30 $\mathrm{mg} / \mathrm{kg}$ durante tres días, inmunoglobulina intravenosa a dosis diarias de 1-2 $\mathrm{mg} / \mathrm{kg}$ por dos a cinco días e, incluso, ha habido tratamientos exitosos con plasmaféresis y otros inmunosupresores como ciclofosfamida, ciclosporina o micofenolato de mofetilo, entre otros ${ }^{(54)}$. No se debe administrar inmunoglobulina intravenosa sin asociarla con esteroides y está contraindicada en pacientes con miocarditis debido a que su viscosidad puede empeorar la función cardiaca ${ }^{(53)}$. También, se debe evitar dar tratamiento antibiótico empírico.

Se debe brindar soporte específico para cada órgano afectado, por ejemplo: diálisis en falla renal aguda; trasplante hepático en falla hepática; diurético, inhibidor de la enzima convertidora de angiotensina y beta bloqueadores, en caso de miocarditis, etc. ${ }^{\left({ }^{54}\right)}$.

El seguimiento posterior a la recuperación clínica y paraclínica, se debe hacer periódicamente debido a que existe la posibilidad de una recaída de la enfermedad y necesidad de aumentar nuevamente la dosis de corticosteroides.

Finalmente, si se descontinúa de manera temprana el medicamento implicado y se trata adecuadamente, gran parte de los pacientes no desarrollarán secuelas. Con el tiempo, otro grupo de pacientes puede desarrollar enfermedades autoinmunitarias de novo y compromiso de órganos, como enfermedades autoinmunitarias de tiroides, lupus eritematoso sistémico, alopecia areata o falla renal con necesidad de hemodiálisis, entre otras ${ }^{(42)}$.

En la tabla 5 se comparan algunas de las características del conjunto del síndrome de Stevens-Johnson y la necrólisis epidérmica tóxica y el síndrome DRESS.

\section{CONCLUSIONES}

Las enfermedades cutáneas potencialmente mortales desencadenadas por medicamentos y, ocasionalmente, por agentes infecciosos, son poco frecuentes. Eso implica que gran parte del personal médico no esté familiarizado con su presentación clínica, y se retrasen el diagnóstico y el retiro del medicamento culpable. El conjunto del síndrome de Stevens-Johnson y la necrólisis epidérmica tóxica es un cuadro con lesiones en placas en diana atípicas, ampollas y signo positivo de Nikolsky, con gran compromiso de las mucosas. Su diagnóstico es principalmente clínico, pues la histopatología no es específica para la enfermedad y retrasa el inicio del tratamiento; se requiere de gran sospecha para poder descontinuar tempranamente el medicamento culpable. Su tratamiento todavía es debatido; 
su pilar es el manejo de la insuficiencia cutánea aguda, y aún no hay consenso sobre el beneficio de la inmunoglobulina intravenosa, de los esteroides sistémicos, ni de los antagonistas del TNF- $\alpha$.

El síndrome DRESS produce poco desprendimiento de la epidermis. A pesar de esto, también se genera insuficiencia cutánea aguda, requiere de manejo en la unidad de cuidados intensivos y, al igual que el conjunto del síndrome de Stevens-Johnson y la necrólisis epidérmica tóxica, requiere de gran sospecha diagnóstica. El principal órgano afectado es el hígado, cuyo daño puede progresar a falla hepática con necesidad de trasplante.

Ambas enfermedades necesitan un seguimiento estricto, usualmente en la unidad de cuidados intensivos o en la de quemados, para mitigar la probabilidad de muerte. Se resalta la necesidad de conocer las enfermedades y los medicamentos desencadenantes, por parte de los médicos de unidades de cuidados intensivos (especialistas y médicos generales), quienes serán los encargados de estabilizar al paciente e impactar en las secuelas.

\section{REFERENCIAS}

1. Roujeau J. Clinical heterogeneity of drug hypersensitivity. Toxicology. 2005;209:123-9.

2. Steven A, Johnson F. A new eruptive fever associated with stomatitis and ophthalmia. Am J Dis Child. 1922;(Case 2).

3. Bohigian GM. The history of Stevens-Johnson Syndrome and a case study. Cent Hist Med Washingt Univ Sch Med St Louis [Internet]. 2015; Available from: http://digitalcommons.wustl.edu/historyofmedicine_presentations/1.

Tabla 5. Cuadro comparativo entre el conjunto del síndrome de Stevens-Johnson y la necrólisis epidérmica tóxica SSJ/NET y síndrome DRESS

\begin{tabular}{|c|c|c|}
\hline & DRESS & SSJ/NET \\
\hline Latencia de inicio de síntomas & 2 a 6 semanas & 1 a 4 semanas \\
\hline \multirow{4}{*}{ Características cutáneas } & Brote maculopapular confluente & $\begin{array}{l}\text { Lesiones en diana atípicas o máculas } \\
\text { purpúricas }\end{array}$ \\
\hline & Dermatitis exfoliativa & Ampollas flácidas \\
\hline & Pústulas & Signo positivo de Nikolsky \\
\hline & Rara la presencia de ampollas & \\
\hline Compromiso de mucosas & $\begin{array}{l}\text { Escaso } \\
\text { Predomina la xerostomía }\end{array}$ & $\begin{array}{l}\text { Acentuado compromiso de mucosas de la } \\
\text { cavidad oral, la conjuntiva o los genitales }\end{array}$ \\
\hline \multirow[b]{2}{*}{ Compromiso visceral } & Es necesario para el diagnóstico. & $\begin{array}{l}\text { Secundario a la deshidratación y al } \\
\text { desprendimiento epitelial }\end{array}$ \\
\hline & $\begin{array}{l}\text { Compromiso directo del sistema hepático, } \\
\text { renal, pulmonar, cardiaco, neurológico o } \\
\text { gastrointestinal }\end{array}$ & $\begin{array}{l}\text { Falla renal aguda, neumonía, elevación de } \\
\text { transaminasas }\end{array}$ \\
\hline Compromiso de ganglios linfáticos & Muy frecuente, es un criterio diagnóstico & Ausente \\
\hline Mortalidad & $10 \%$ & $<6 \%$ a $45 \%$ \\
\hline \multirow[b]{2}{*}{ Tratamiento } & $\begin{array}{l}\text { Tratamiento de referencia: } \\
\text { corticosteroides sistémicos y manejo de } \\
\text { sostenimiento }\end{array}$ & $\begin{array}{l}\text { Tratamiento de referencia: manejo de } \\
\text { sostenimiento }\end{array}$ \\
\hline & $\begin{array}{l}\text { Otros con buena evidencia: } \\
\text { plasmaféresis, ciclofosfamida, } \\
\text { ciclosporina y micofenolato de mofetilo }\end{array}$ & $\begin{array}{l}\text { Otros con evidencia pendiente: } \\
\text { corticosteroides sistémicos, } \\
\text { inmunoglobulina intravenosa, } \\
\text { ciclosporina y anti-TNF-a }\end{array}$ \\
\hline
\end{tabular}

SSJ/NET: síndrome de Stevens-Johnson y necrólisis epidérmica tóxica 
4. Lyell A. Toxic epidermal necrolysis: An eruption resembling scalding of the skin. Br J Dermatol. 1956;68:355-61.

5. Sun J, Liu J, Gong QL, Ding GZ, Ma LW, Zhang LC, et al. Stevens-Johnson Syndrome and toxic epidermal necrolysis: A multi-aspect comparative 7-year study from the People's Republic of China. Drug Des Devel Ther. 2014;8:2539-47.

6. Harr T, French LE. Severe cutaneous adverse reactions: Acute generalized exanthematous pustulosis, toxic epidermal necrolysis and Stevens-Johnson syndrome. Med Clin North Am. 2010;94:727-42.

7. Lin YF, Yang CH, Sindy H, Lin JY, Hui CYR, Tsai YC, et al. Severe cutaneous adverse reactions related to systemic antibiotics. Clin Infect Dis. 2014;58:1377-85.

8. Mahar PD, Wasiak J, Hii B, Cleland H, Watters DA, Gin D, et al. A systematic review of the management and outcome of toxic epidermal necrolysis treated in burns centres. Burns. 2014;40:1245-54.

9. Badia M, Serviá L, Casanova JM, Montserrat N, Vilanova J, Vicario E, et al. Classification of dermatological disorders in critical care patients: A prospective observational study. J Crit Care. 2013;28:220.e1-220.e8.

10. Peña-Hoyos ÁA. Enfermedades dermatológicas de pacientes hospitalizados en cuidados intensivos, Hospital de La Samaritana. Bogotá: Universidad Nacional de Colombia; 2016.

11. Victoria-Chaparro J. Eritema multiforme. In: Restrepo-Castaño A, Franco RS, editors. Dermatología. Octava edición. Medellín, Colombia: Corporación para Investigaciones Biológicas; 2017. p. 129-37.

12. Walicka M, Majsterek M, Rakowska A, Słowińska M, Sicińska J, Góralska B, et al. Mycoplasma pneumoniae-induced pneumonia with StevensJohnson syndrome of acute atypical course. Polish Arch Intern Med. 2008;118:449-53.

13. Domínguez-Borgua A, González I, Morales L, Martinez-Carrillo MF, Palacios P. Toxic epidermal necrolysis and development of liver abscesses. Gac Med Mex. 2015;151:512-8.

14. Uzun R, Yalcin AD, Celik B, Bulut T, Yalcin AN. Levofloxacin induced toxic epidermal necrolysis: Successful therapy with omalizumab (antiIgE) and pulse prednisolone. Am J Case Rep. 2016;17:666-71.

15. Yang CY, Dao RL, Lee TJ, Lu CW, Yang CH, Hung SI, et al. Severe cutaneous adverse reactions to anti- epileptic drugs in Asians. Neurology. 2011;77:202533.

16. Godhwani N, Bahna SL. Antiepilepsy drugs and the immune system. Ann Allergy Asthma Immunol. 2016;117:634-40.

17. Harr T, French LE. Toxic epidermal necrolysis and Stevens Johnson syndrome. Orphanet J Rare Dis. 2010;5:1-11.

18. Changela A, Javaiya H, Rickenback K, Elnawawi A, Changela K. Toxic epidermal necrolyis after vancomycin use: A case report and discussion of management. Am J Ther. 2013;20:223-5.

19. Harris V, Jackson C, Cooper A. Review of toxic epidermal necrolysis. Int J Mol Sci. 2016;17:1-11.

20. Lim VM, Do A, Berger TG, Nguyen AH, Deweese J, Malone JD, et al. A decade of burn unit experience with Stevens-Johnson syndrome/toxic epidermal necrolysis: Clinical pathological diagnosis and risk factor awareness. Burns. 2016;42:836-43.

21. Brickel N, Shaikh H, Kirkham A, Davies G, Chalker M, Yoshida P. Collaboration in pharmacovigilance: lamotrigine and fatal severe cutaneous adverse reactions - a review of spontaneous reports. Ther Clin Risk Manag. 2017;13:897-903.

22. Dodiuk-Gad RP, Chung WH, Valeyrie-Allanore L, Shear NH. Stevens-Johnson syndrome and toxic epidermal necrolysis: An update. Am J Clin Dermatol. 2015;16:475-93.

23. Galindo-Díaz LF, Díaz-Guío DA. Insuficiencia cutánea aguda. En: Díaz-Guío DA, editor. Cuarta edición. Armenia, Quindío: Publicaciones Vital Care Cuidado Crítico; 2013. p. 478-86.

24. Paradisi A, Abeni D, Bergamo F, Ricci F, Didona D, Didona B. Etanercept therapy for toxic epidermal necrolysis. J Am Acad Dermatol. 2014;71:278-83.

25. Yang LP, Zhang AL, Wang DD, Ke HX, Cheng Q, Wang C. Stevens-Johnson syndrome induced by the cross-reactivity between teicoplanin and vancomycin. J Clin Pharm Ther. 2014;39:442-5.

26. Arshad F, Bhat TS, Lone AR. Docetaxel induced Lyell's syndrome: A rare life threatening cause of dermatitis medicamentosas. J Cancer Res Ther. 2014;10:742-4.

27. Creamer D, Walsh SA, Dziewulski P, Exton LS, Lee HY, Dart JKG, et al. UK guidelines for the management of Stevens-Johnson syndrome/toxic epidermal necrolysis in adults 2016. J Plast Reconstr Aesthetic Surg. 2016;69:e119-227.

28. Yamane Y, Matsukura S, Watanabe Y, Yamaguchi Y, Nakamura K, Kambara T, et al. Retrospective analysis of Stevens-Johnson syndrome and toxic 
epidermal necrolysis in 87 Japanese patients Treatment and outcome. Allergol Int. 2016;65:7481.

29. De Prost N, Mekontso-Dessap A, Valeyrie-Allanore L, van Nhieu JT, Duong TA, Chosidow O, et al. Acute respiratory failure in patients with toxic epidermal necrolysis: Clinical features and factors associated with mechanical ventilation. Crit Care Med. 2014;42:118-28.

30. Bastuji-Garin S, Fouchard N, Bertocchi M, Roujeau JC, Revuz J, Wolkenstein P. Scorten: A severity-ofillness score for toxic epidermal necrolysis. J Invest Dermatol. 2000;115:149-53.

31. Seczynska B, Nowak I, Sega A, Kozka M, Wodkowski M, Szczeklik W. Supportive therapy for a patient with toxic epidermal necrolysis undergoing plasmapheresis. Crit Care Nurse. 2013;33:2638.

32. Schneider JA, Cohen PR. Stevens-Johnson syndrome and toxic epidermal necrolysis: A concise review with a comprehensive summary of therapeutic interventions emphasizing supportive measures. Adv Ther. 2017;34:1235-44.

33. World Health Organization. WHO's cancer pain ladder for adults [Internet]; 2013. Fecha de consulta: 18 de febrero de 2018. Disponible en: http:// www.who.int/cancer/palliative/painladder/en/.

34. Sekula P, Dunant A, Mockenhaupt M, Naldi L, Bouwes Bavinck JN, Halevy S, et al. Comprehensive survival analysis of a cohort of patients with Stevens-Johnson syndrome and toxic epidermal necrolysis. J Invest Dermatol. 2013;133:1197204.

35. Wolf R, Davidovici B. Severe cutaneous adverse drug reactions: Who should treat, where and how? Facts and controversies. Clin Dermatol. 2010;28:344-8.

36. Paquet P, Piérard GE. New insights in toxic epidermal necrolysis ( Lyell' syndrome). Drug Saf. 2010;33:189-212.

37. Saavedra C, Cárdenas P, Castellanos H, Contreras $\mathrm{K}$, Castro JR. Cephazolin-induced toxic epidermal necrolysis treated with intravenous immunoglobulin and N-acetylcysteine. Case Reports Immunol. 2012;2012:1-4.

38. Husain Z, Reddy BY, Schwartz RA. DRESS syndrome: Part I. Clinical perspectives. J Am Acad Dermatol. 2013;68:693.e1-693.e14.

39. Shiohara T, Kano Y. Drug reaction with eosinophilia and systemic symptoms (DRESS): Incidence, pathogenesis and management. Expert Opin Drug Saf. 2017;16:139-47.
40. EL omairi N, Abourazzak S, Chaouki S, Atmani S, Hida M. Drug reaction with eosinophilia and systemic symptom (DRESS) induced by carbamazepine: Case report and literature review. PanAfrican Med J. 2014;8688:1-4.

41. Kimmoun A, Dubois E, Perez P, Barbaud A, Levy B. Shock state: An unrecognized and underestimated presentation of drug reaction with eosinophilia and systemic symptoms. Shock. 2013;40:387-91.

42. Chen YC, Cho YT, Chang CY, Chu CY. Drug reaction with eosinophilia and systemic symptoms: A drug-induced hypersensitivity syndrome with variable clinical features. Dermatologica Sin. 2013;31:196-204.

43. Błaszczyk B, Lasoń W, Czuczwar SJ. Antiepileptic drugs and adverse skin reactions: An update. Pharmacol Reports. 2015;67:426-34.

44. Sasidharanpillai S, Riyaz N, Rajan U, Binitha M, Khader A, Reena Mariyath O, et al. Drug reaction with eosinophilia and systemic symptoms: Observations from a tertiary care institution. Indian J Dermatol Venereol Leprol. 2014;80:221-8.

45. Ichai P, Laurent-Bellue A, Saliba F, Moreau D, Besch C, Francoz C, et al. Acute liver failure/injury related to drug reaction with eosinophilia and systemic symptoms: Outcomes and prognostic factors. Transplantation. 2017;101:1830-7.

46. Hamm RL. Drug-hypersensitivity syndrome: Diagnosis and treatment. J Am Coll Clin Wound Spec. 2011;3:77-81.

47. Spriet S, Banks TA. Drug reaction with eosinophilia and systemic symptoms syndrome. Allergy Asthma Proc. 2015;36:501-5.

48. Miyazu D, Kodama N, Yamashita D, Tanaka H, Inoue $\mathrm{S}$, Imakyure $\mathrm{O}$, et al. DRESS syndrome caused by cross-reactivity between vancomycin and subsequent teicoplanin administration: A case report. Am J Case Rep. 2016;17:625-31.

49. Nam YH, Park MR, Nam HJ, Lee SK, Kim KH, Roh MS, et al. Drug reaction with eosinophilia and systemic symptoms syndrome is not uncommon and shows better clinical outcome than generally recognised. Allergol Immunopathol (Madr). 2015;43:19-24.

50. Moriceau F, Prothet J, Blaise BJ, Ben Said B, Page $\mathrm{M}$, Ber CE, et al. DRESS syndrome in the ICU: When a patient Is treated with multiple drugs. Case Reports Crit Care. 2016;2016:1-4.

51. Criado PR, Criado RFJ, Avancini J de M, Santi CG. A case of drug reaction with eosinophilia and systemic symptoms. An Bras Dermatol. 2012;87:43549. 
52. Borok J, Hau J, Worswick S. Adult with morbilliform rash and tattoo bullae. Dermatol Online J. 2016;22:3-7.

53. Thongsri T, Chularojanamontri L, Pichler W j. Cardiac involvement in DRESS syndrome. Asian Pacific J Allergy Immunol. 2017;35:3-10.

54. Husain Z, Reddy BY, Schwartz RA. DRESS syndrome: Part II. Management and therapeutics. J Am Acad Dermatol. 2013;68:709.e1-709.e9.

55. Ocampo-Garza J, Ocampo-Garza SS, MartínezVillarreal JD, Barbosa-Moreno LE, Guerrero-González GA, Ocampo-Candiani J. Reacción por drogas con eosinofilia y síntomas sistémicos (síndrome de DRESS). Estudio retrospectivo de nueve casos. Rev Med Chil. 2015;143:577-83. 